\title{
Dynamical models for sand ripples beneath surface waves
}

\section{Andersen, Ken Haste; Chabanol, M.-L.; v. Hecke, M.}

\section{Published in:}

Physical Review E. Statistical, Nonlinear, and Soft Matter Physics

Link to article, DOI:

10.1103/PhysRevE.63.066308

Publication date:

2001

Document Version

Publisher's PDF, also known as Version of record

Link back to DTU Orbit

Citation (APA):

Andersen, K. H., Chabanol, M-L., \& v. Hecke, M. (2001). Dynamical models for sand ripples beneath surface waves. Physical Review E. Statistical, Nonlinear, and Soft Matter Physics, 63(6), 066308.

https://doi.org/10.1103/PhysRevE.63.066308

\section{General rights}

Copyright and moral rights for the publications made accessible in the public portal are retained by the authors and/or other copyright owners and it is a condition of accessing publications that users recognise and abide by the legal requirements associated with these rights.

- Users may download and print one copy of any publication from the public portal for the purpose of private study or research.

- You may not further distribute the material or use it for any profit-making activity or commercial gain

- You may freely distribute the URL identifying the publication in the public portal

If you believe that this document breaches copyright please contact us providing details, and we will remove access to the work immediately and investigate your claim 


\title{
Dynamical models for sand ripples beneath surface waves
}

\author{
Ken Haste Andersen, ${ }^{1,2,3}$ Marie-Line Chabanol, ${ }^{1,4}$ and Martin van Hecke ${ }^{1,5}$ \\ ${ }^{1}$ Center for Chaos and Turbulence Studies, Niels Bohr Institute, Blegdamsvej 17, 2100 Copenhagen , Denmark \\ ${ }^{2}$ Institute of Hydraulic Research and Water Resources, Technical University of Denmark, 2800 Lyngby, Denmark \\ ${ }^{3}$ Dipartimento di Fisica, Universita degli Studi di Roma La Sapienza, Piazzale Aldo Moro 2, I-00185 Roma, Italy \\ ${ }^{4}$ Institut Fourier, 100 rue des Mathématiques, Domaine Universitaire, 38400 Saint Martin d'Hères, France \\ ${ }^{5}$ Max-Planck-Institut für Physik Komplexer Systeme, Nöthnitzer Strasse 3801187 Dresden, Germany
}

(Received 30 March 2000; revised manuscript received 26 February 2001; published 29 May 2001)

\begin{abstract}
We introduce order parameter models for describing the dynamics of sand ripple patterns under oscillatory flow. A crucial ingredient of these models is the mass transport between adjacent ripples, which we obtain from detailed numerical simulations for a range of ripple sizes. Using this mass transport function, our models predict the existence of a stable band of wave numbers limited by secondary instabilities. Small ripples coarsen in our models and this process leads to a sharply selected final wave number, in agreement with experimental observations.
\end{abstract}

DOI: 10.1103/PhysRevE.63.066308

PACS number(s): 45.70.Qj, 47.32.Cc, 47.27.Nz, 45.50.Jf

\section{INTRODUCTION}

When a flat surface of sand is exposed to the flow of air or water, patterns known as ripples, dunes, sand waves and draas are formed [1-6]. Here we focus on the so-called vortex ripples [1] (Fig. 1) that are created by oscillatory fluid flow, e.g., beneath surface waves. Ripples are of interest to coastal engineers since their properties determine the friction of the flow in the coastal region [7-10], the dissipation of surface waves [11] and the net sediment transport over the ripples [12]. More recently ripples have attracted the attention of physicists interested in nonequilibrium systems $[2,4,13-20]$.

The physics underlying sand ripple formation involves the interaction between the turbulent fluid flow and a granular medium, and is therefore extremely complex. A description of the pattern forming aspects is hindered by the strong nonlinearity of the fully developed ripples due to the subcritical nature of the initial bifurcation from a flat bed. Previous theoretical studies of this initial bifurcation [21-24] have described the onset of ripple formation. Vortex ripple pattern formation occurs, however, far from equilibrium: typical wavelengths of fully developed ripples can be a factor 5 larger than those predicted by (weakly nonlinear) analysis [24].

In this paper we will discuss the pattern forming aspects of fully developed vortex ripples. Many of the problems associated with the complicated underlying phenomenology can be circumvented by noting that the sizes of the ripples are the most relevant parameter for determination of their dynamics; further details of their shapes are not important. Dynamical equations for the evolution of the ripples can then be constructed once the mass exchange between ripples of certain sizes is known. We base our expression for this mass exchange on detailed numerical simulations of the flow and sand transport over vortex ripples (see below), hence going beyond a pure "toy-model" approach. As far as we are aware, the model presented here is the first to capture both instabilities and coarsening of fully developed vortex ripples.

The outline of the paper is as follows. We start with a brief description of the main phenomenology of ripples in Sec. II. Although the amplitude of the fluid oscillations determines the length of the ripples, a dimensional analysis (Sec. II A) reveals that the most relevant dimensionless control parameter is the Shields parameter that characterizes stress at the sandy surface. We discuss our numerical simulations of the mass exchange between vortex ripples in Sec. II B. Section III is devoted to the formulation of simple ripple models in one-dimensional geometries. The linear stability of these models is performed in Sec. III B, and the coarsening and selection of the final ripple patterns starting from random initial conditions is discussed in Sec. III D. In Sec. IV we extend our model to two dimensions and discuss the impact of defect motion on the selection of the final two-dimensional pattern.

\section{VORTEX RIPPLES}

Following the much earlier work of Ayrton [25], the study of vortex ripples was taken up again by Bagnold in 1946 [3]. In this seminal study, Bagnold distinguished between rolling grain ripples and vortex ripples. The former are generated when starting from an unstable flat bed [5] and consist of small triangular ridges separated by a comparatively long stretch of flat bed. These rolling grain ripples grow and coarsen to become vortex ripples with no flat bed between them. Here the flow is dominated by separation bubbles (vortices) on the lee sides of the fully developed ripples. We will concentrate on these fully developed vortex ripples, since recent studies have confirmed $[19,20]$ that rolling grain ripples essentially constitute a transient.

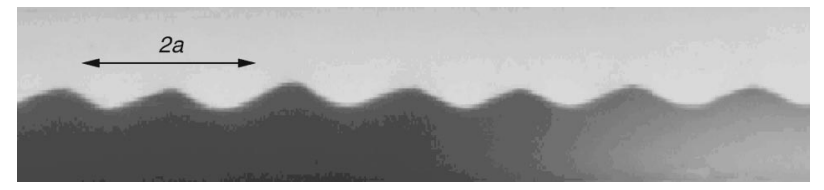

FIG. 1. Side view of a vortex ripple pattern under oscillatory flow in a long, slender channel (courtesy of J.L. Hansen). The arrow indicates the amplitude of the fluid oscillations in the bulk. 
Many experiments have studied the average wavelength of fully developed ripples [26-29] as a function of, e.g., the amplitude and frequency of the fluid motion. It appears that the (dimensional) length of the ripples $\lambda_{d i m}$ is proportional to the amplitude of the oscillatory flow $a$, and roughly independent of its frequency. Estimates in the literature of the proportionality constant $\lambda_{\operatorname{dim}} / a$ range from one to two, with a preference for values around 1.3 (see Fig. 8 in [27]).

Recently the ripples have also been studied from the view point of pattern formation. Both Scherer et al. [20] and Stegner and Wesfreid [19] studied a one-dimensional annular system in which the conservation of sand is guaranteed. Stegner and Wesfried [19] observed strong hysteresis when the driving amplitude of fully developed ripples was ramped up and down: an increase in the amplitude of the driving yielded larger ripples, while for a decrease, the ripples did not change length. Lofquist also [7] observed hysteretic behavior, but in this case the ripples were initially stable for both an increase or decrease of the driving amplitude [7]. Hysteresis of the ripples was also observed in a recent set of field measurements [29].

\section{A. Dimensional analysis and setup of the problem}

Ripples are governed by a large number of dimensional parameters that characterize fluid flow and sand. We will show that while in general three dimensionless parameters (density ratio of fluid and sand grains $s$, settling velocity $w_{s}$, and maximum Shields parameter $\theta_{\max }$ ) characterize the system, for the case of interest here (sand-water systems in the regime where suspension is unimportant) the only free parameter is the Shields parameter.

Ripple formation is driven by an oscillatory fluid motion with amplitude $a$ and angular frequency $\omega$. The Reynolds number Re for this situation is $a^{2} \omega / \nu$, where $\nu$ is the fluid viscosity. For water in a typical experimental situation ( $a$ $\left.=5 \mathrm{~cm}, \omega=3 \mathrm{~s}^{-1}\right), \operatorname{Re}$ is of order $10^{3}$, and the flow is turbulent. Therefore, large scale flow structures such as separation bubbles are independent of the Reynolds number and hence viscosity. For turbulent flow, the roughness of the bed is of minor importance as long as the typical grain sizes are much smaller than $a$. The only relevant length scale is then $a$, which we use to define the nondimensional ripple length as $\lambda=\lambda_{\text {dim }} / a$. The large scale flow is then completely specified by the boundary conditions, i.e., the shape of the ripples.

The sand introduces four new dimensional parameters into the problem. These are, respectively, the density of water $\rho_{w}$ and sand $\rho_{s}$, the median diameter of the grains $d$ and gravity $g$. From these we form the following three nondimensional parameters:

$$
s=\frac{\rho_{s}}{\rho_{w}}, \quad w_{s}=\frac{w_{s . d i m}}{a \omega}, \quad \theta=\frac{\tau_{\text {bed }}}{\rho_{w}(s-1) g d},
$$

The relative density of the grains $s$ has a value of 2.65 for quartz sand in water. The settling velocity $\omega_{s}$ characterizes the amount of sand kept in suspension; here we assume a regime where the settling velocity is large $\left(w_{s} \gtrsim 0.15\right)$ such that suspension is not important. This leaves us with the last parameter $\theta$, which is known as the Shields parameter. The Shields parameter expresses the ratio between the drag and gravitational forces on a single grain and depends on the shear stress $\tau_{\text {bed }}(x, t)$, which varies with time and along the profile of the ripple. Following [30] we propose to use the maximum shear stress on a flat bed $\tau_{\max }$ to characterize the flow. For laminar flow $\tau_{\max }$ can be found exactly from the solution of Stokes' second problem [31]. For turbulent conditions, which prevail here, an analytical expression does not exist. We will follow coastal engineers in using an empirical relation for the maximum shear stress [32]:

$$
\tau_{\max }=0.02 \rho_{w}\left(\frac{a}{k_{N}}\right)^{-0.25}(a \omega)^{2} .
$$

Note that the instantaneous Shields parameter on a rippled bed $\theta(x, t)$ can be several times larger than $\theta_{\max }$.

The transport of sand $q$ takes place in a thin layer above the bed, the so-called bed load layer (for an introduction to sediment transport see Chap. 7 in Ref. [32]). The nondimensionalized flux of sand $\phi \equiv q / \sqrt{g(s-1) d^{3}}$ in the bed load layer is a function of the local Shields parameter and can be modeled as

$$
\phi=\alpha\left(\theta-\theta_{c}\right)^{\beta} .
$$

When $\theta(x, t)$ smaller than a critical value $\theta_{c}$ for all $x$, which for turbulent boundary layers is approximately 0.06 [32], sand grains do not move and the ripple profile freezes. The constants $\alpha$ and $\beta$ have been determined empirically by Meyer-Peter and Müller [33] to be approximately $\alpha=8$ and $\beta=1.5$, which are in good agreement with theoretical estimates $[34,35]$. The formation and the dynamical properties of the ripples are mainly determined by the fluid flow, so the exact values of the constants $\alpha$ and $\beta$ together with the detailed form of Eq. (3), turn out to be relatively unimportant for the content of this work.

\section{B. Numerical studies and mass transport}

The computational model that we have developed to study the ripples calculates turbulent fluid flow over ripples based on the standard $k-\omega$ turbulence model [36,37]. Once this flow is known, the sediment transport, which is governed by the shear stress on the bed, can be calculated from Eq. (3).

In Fig. 2 we show some results for the flow and the nondimensionalized shear stress $\phi$ for $\lambda=1.15$. We see that there are two mechanisms that generate the shear stress on the bed, namely, the converging flow on the "wind" side of the ripple (here left) and the separation bubble formed in the lee (right) side. Typically, these stresses are several times stronger than the stresses on a flat bed. The separation bubble, where the flow near the bed is directed opposite to the mean flow direction, is clearly visible. This bubble moves out into the trough of the ripple $\left(\omega t=90^{\circ}\right)$, where it stays $\left(\omega t=150^{\circ}\right)$ until it is thrown over the crest as the flow reverses.

The shear stresses are uphill on both sides of the ripple, and consequently sand is transported from the trough toward 

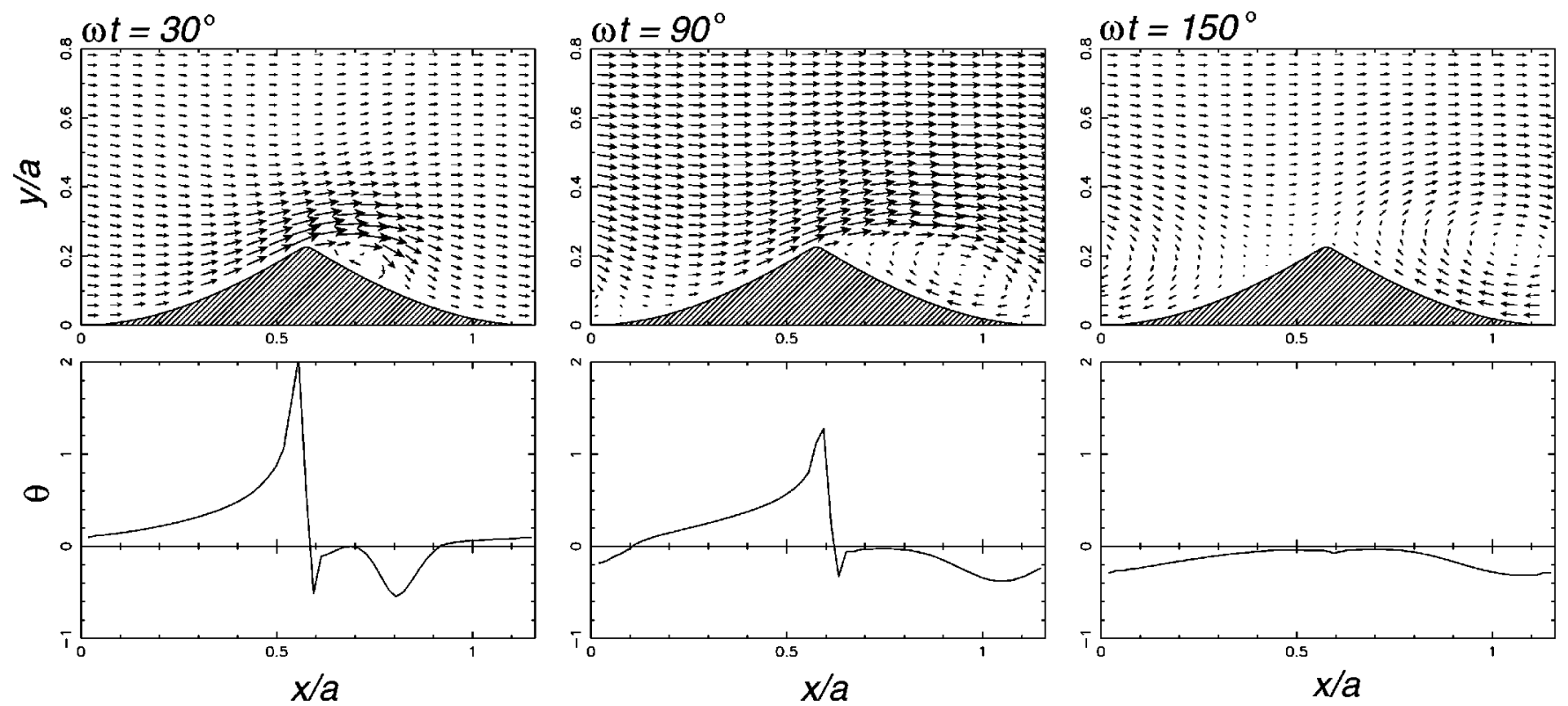

FIG. 2. The flow over a ripple at three instants in the first half of the wave period. The system consists of a single ripple in a system with periodic boundary conditions and length $\lambda=1.15$. The bottom three figures show the spatial profile of the Shields parameter on the bed at the corresponding times. For this case $\theta_{\max }=0.13$.

the crest; the result is a steepening of the ripple profile. This steepening continues until the slopes of the ripple reach the angle of repose, where avalanches limit the growth of the ripple slopes. As a consequence, most slopes of the fully developed ripples are close to the angle of repose. These fully developed ripples are thus approximately triangular, joined by smooth troughs, which is also evident from experiments [19].

Ripples interact by exchanging sand between their neighbors over the troughs. The amount of this mass flow is closely connected to the extension and strength of the separation bubble. We have studied this mass transport as a function of the nondimensional ripple size $\lambda$. In Fig. 3 we show the net sediment transport during the first half wave period, for short $(\lambda=0.6)$, medium $(\lambda=1.0)$ and large ripples $(\lambda$

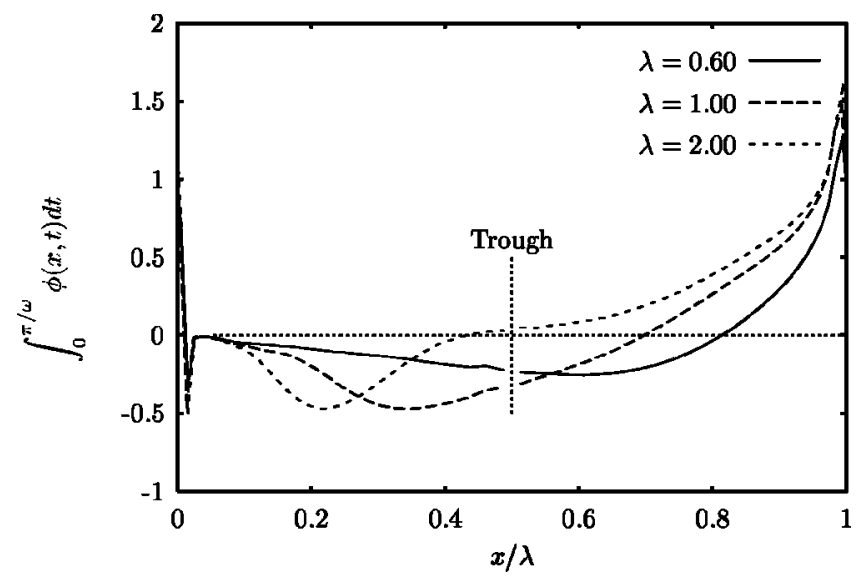

FIG. 3. The spatial profile of the net mass transport in the first half of the wave period $\left[\int_{0}^{\pi / \omega} \phi(x, t) d t\right]$ for ripples of length $\lambda$ $=0.6,1.0$, and 2.0. Note that the ripple trough is located in the middle of the figure. $\left(\theta_{\max }=0.13\right)$.
$=2.0$ ). For short ripples the separation bubble almost covers the space between the two ripple crests, but it is not very strong, giving rise to a small transport. For long ripples, the separation bubble does not reach over the trough, again giving only a small mass exchange between adjacent ripples. Most mass is exchanged for medium sized ripples, where the separation bubble is both strong and reaches over the trough.

We define $f$ as the amount of sand transported over the trough during the first half wave period

$$
f(\lambda)=-\int_{0}^{\pi / \omega} \phi\left(x_{t r}, t\right) d t
$$

where $x_{t r}$ is the position of the trough. The minus sign is simply related to the fact that the fluid and mass flows have opposite directions during each half period; here we wish to have a positive $f(\lambda)$.

The rescaled mass exchange $f(\lambda) / f(\lambda=1.0)$ is shown in Fig. 4 for values of $\theta_{\max }$ ranging from 0.075 to 0.75 . The rescaled graphs of the mass exchange (i) collapse in good approximation and (ii) have a single maximum around $\lambda$ $=1.0$. In our model, developed in Sec. III below, we will incorporate these two properties. If the critical Shields parameter had been 0 , the rescaling factor $f(\lambda=1.0)$ would have been proportional to $\theta_{\max }^{1.5}$. That this is almost, but not exactly, the case is seen in the inset in Fig. 4.

\section{DISCRETE MODELS FOR ONE-DIMENSIONAL RIPPLES}

In this section we will introduce and study simple models for ripples in one-dimensional geometries. We assume that the angles of the ripple slopes are fixed, so that the only degrees of freedom are the lengths of their left and right slopes. Two different versions of the model will be de- 


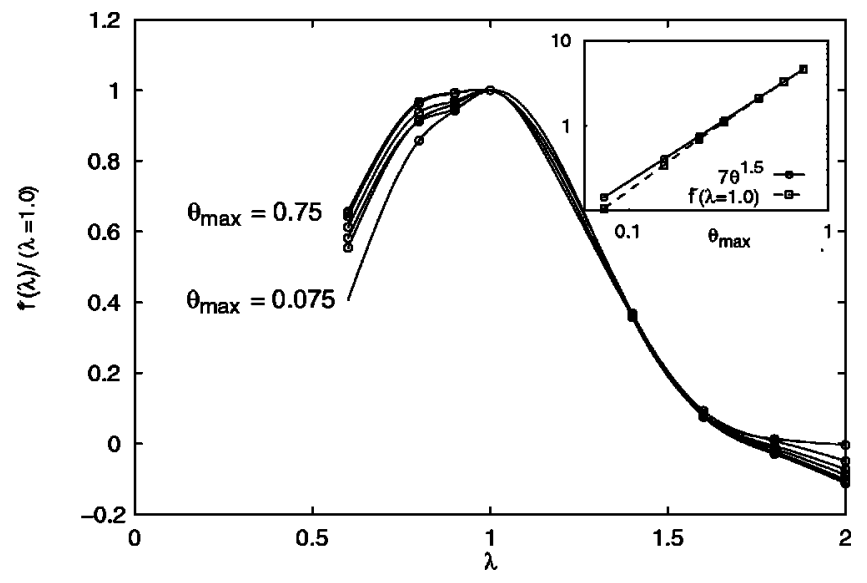

FIG. 4. $f(\lambda)$ for $\theta_{\max }=0.075,0.15,0.23,0.30,0.45,0.60$ and 0.75 , scaled with $f(\lambda=1.0)$. The inset show $7 \theta_{\text {max }}^{1.5}$ (full line) and $f(\lambda=1.0)$ (dashed line) in double log scaling.

scribed. In the simplest case we only take the total ripple sizes $\lambda_{i}$ into account (see Fig. 5). The ensuing "minimal model" 'is formulated in Sec. III A, and is analyzed theoretically in Sec. III B. A more refined model that takes the lengths of left and right slopes into account is presented in Sec. III C (see Fig. 6), and numerical simulations of this model are presented in Sec. III D.

\section{A. Minimal model}

In this model ripples are triangular and symmetric and characterized by their length $\lambda_{i}$. We will now determine the mass transfer between two ripples with lengths $\lambda_{1}$ and $\lambda_{2}$ (Fig. 5) from the information that we have for the mass transfer between equal ripples. When $\lambda_{1}$ and $\lambda_{2}$ are approximately equal, one expects the size and strength of the separation bubble emanating from the crest of ripple 1 to be independent of the size of ripple 2. This is our central assumption: the mass transport during a half period only depends on the size of the ripple that creates the separation bubble.

Let us denote the first half period of the driving, when the flow is from left to right, by a subscript I, and the second half by II. Under our assumption stated above, we obtain: $\Delta m_{\mathrm{I}}$ $=f\left(\lambda_{1}\right)$ and $\Delta m_{\mathrm{II}}=-f\left(\lambda_{2}\right)$, where $\Delta m_{\mathrm{I}}$ denotes the change in the mass of ripple 1 in the first half period. During each half period, the amount of mass transported is small in com-

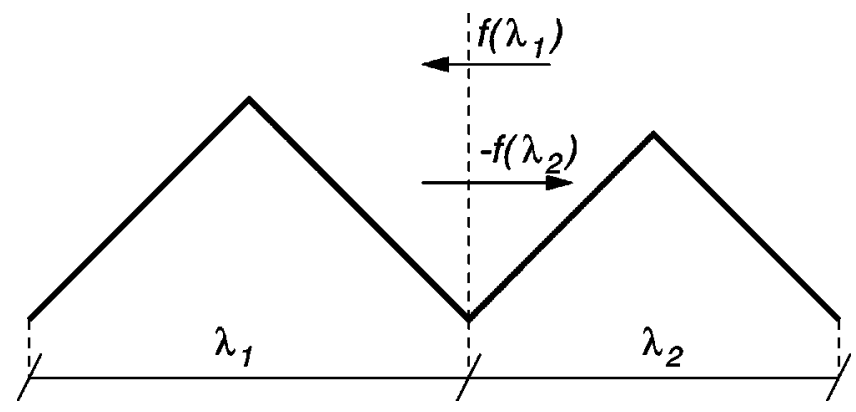

FIG. 5. An example of how the transport in the trough between two ripples is constructed as the transport in the two half periods.

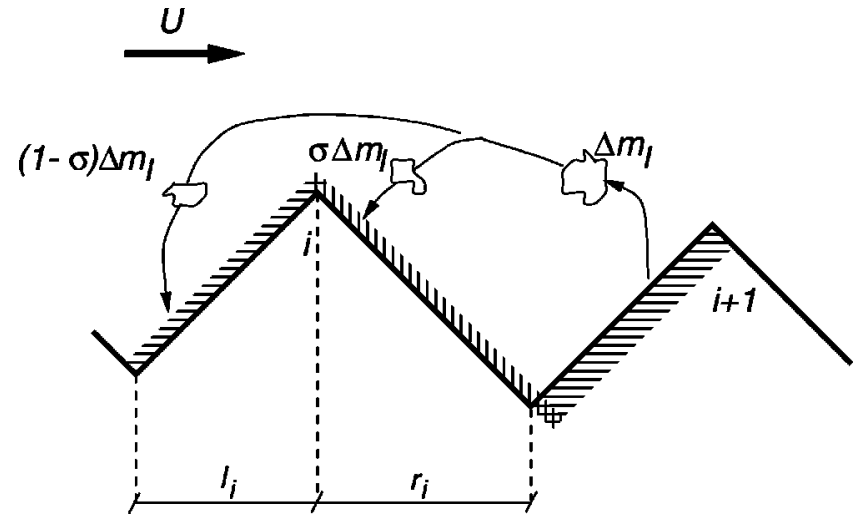

FIG. 6. A sketch of the ripple profile with the triangular ripples and the quantities used to describe the ripples. Note that the ripples do not need to be symmetric. Also shown is the exchange of sediment from the right ripple to the left ripple during the first half wave period.

parison to the mass of a single ripple. We therefore can neglect changes in the ripple shapes during a half period, and obtain the mass flow during a full period, $\Delta m$, by simply adding up the half period mass flows

$$
\Delta m=f\left(\lambda_{1}\right)-f\left(\lambda_{2}\right)
$$

Clearly Eq. (5) can be extended to the case of a row of ripples. Then the mass flow to ripple $i, \Delta m_{i}$, is due to interactions with both ripple $i-1$ and $i+1: \Delta m_{i}=2 f\left(\lambda_{i}\right)$ $-f\left(\lambda_{i+1}\right)-f\left(\lambda_{i-1}\right)$.

To close the equations we need to relate the mass flow to a change in the size of the ripples. Since the mass-flow is small, it is reasonable to assume that the change in ripple size is linear in the mass transport. The greatest simplification is obtained if we assume all ripples to be of near equal size, so that the ratio $\Delta m / \Delta \lambda$ is equal for all ripples. Taking the continuum time limit and rescaling time to absorb a proportionality constant we obtain

$$
d \lambda_{i} / d t=-f\left(\lambda_{i-1}\right)+2 f\left(\lambda_{i}\right)-f\left(\lambda_{i+1}\right) .
$$

The total length of a system of ripples evolving according to Eq. (6) is conserved, but the total mass is not; we will discuss this further in Sec. III C.

Finally, we supplement the model with an annihilation rule that removes ripples that have shrunk to size zero, and a creation rule, which adds ripples in the troughs between ripples of sizes larger than a certain length $\lambda_{\max }$ that will be specified in the next section.

\section{B. Equilibria and stability}

There are three types of equilibria in the minimal model (6): (i) Homogeneous states where all $\lambda$ 's are equal; (ii) 'Period two" states, for which $f\left(\lambda_{i}\right)=f\left(\lambda_{i+1}\right)$ but $\lambda_{i} \neq \lambda_{i+1}$ (see Fig. 16 in [20] for similar states); and (iii) More complicated equilibria constructed by arbitrary juxtapositions of ripples of lengths $\lambda_{a}$ or $\lambda_{b}$ when $f\left(\lambda_{a}\right)=f\left(\lambda_{b}\right)$.

The linear stability of the homogeneous state follows from setting $\lambda_{i}=\lambda_{e q}+\delta_{i}$ and linearizing Eq. (6) 


$$
d \delta_{i} / d t=-f^{\prime}\left(\lambda_{e q}\right)\left(\delta_{i-1}-2 \delta_{i}+\delta_{i+1}\right)
$$

This is the linear stability equation for the space-discretized diffusion equation, with diffusion coefficient $-f^{\prime}\left(\lambda_{e q}\right)$, and the sign of $f^{\prime}$ will be important. As we demonstrated in Sec. II $\mathrm{B}$, the mass transport $f$ displays a single maximum as a function of $\lambda$ at a value that we will refer to as $\lambda_{\text {min }}$. When $\lambda_{e q}$ is larger (smaller) than $\lambda_{\min },-f^{\prime}\left(\lambda_{e q}\right)$ is positive (negative) and the pattern is stable (unstable). Hence the smallest possible stable wavelength is $\lambda_{\min }$, where $f$ has a maximum. This instability can be seen directly from the mass transport: when we inspect two unequal adjacent ripples with sizes larger than $\lambda_{\min }$ we obtain from Eq. (5) that mass will flow from the larger to the smaller ripple, hence leading to a stable equilibrium, while if their sizes are smaller than $\lambda_{\min }$ mass flows from the smaller to the larger ripple, leading to an instability.

An additional instability occurs for large ripples when their troughs lie outside the separation zone (see $\lambda=2.0$ in Fig. 3); in this case the flow creates new ripples in the troughs. This instability has been observed in experiments $[4,7]$ and also in our numerical studies [37]. This instability is consistent with our models when we assume that $f$ is defined for arbitrary small ripples. For a homogeneous pattern of large ripples where $f\left(\lambda_{e q}\right)<f(0)$, infinitesimal ripples inserted between the large ripples will gain mass and grow, and the maximum value $\lambda_{\max }$ where homogeneous patterns are stable, is given by $f\left(\lambda_{\max }\right)=f(0)$. This is the motivation for having a creation rule in the model.

The period 2 and more complicated equilibrium states can be shown to be unstable in our framework [38]. Thus our model illustrates an important consequence of the shape of the mass exchange function. There is a band of wavelengths for which ripple patterns are stable; outside this band, short wavelength instabilities occur.

\section{Refined model}

Both our numerical studies and experiments [19] frequently display ripples that are asymmetric during their evolution (although, on average, they are not). We extend the minimal model from the previous section to allow for asymmetric ripples by characterizing the ripples by the length of both their left $\left(l_{i}\right)$ and right $\left(r_{i}\right)$ slopes; obviously $\lambda_{i}=l_{i}$ $+r_{i}$ (see Fig. 6). In addition, such a model can be tuned so as to conserve mass.

As before we assume that the lee side of ripples determines the size of the separation bubbles. During the first half period the bubble takes $\Delta m_{\mathrm{I}}$ mass from the left slope of ripple $i+1$, and transports this mass to ripple $i$; the ratio between the mass deposited on the left and right slopes of ripple $i$ is given by a parameter $\sigma$ that we always fix at a value of 0.5 (see Fig. 6). The mass flow in the first half period is therefore

$$
\begin{gathered}
\Delta m_{l i . I}=-f\left(2 r_{i-1}\right)+(1-\sigma) f\left(2 r_{i}\right), \\
\Delta m_{r i . I}=\sigma f\left(2 r_{i}\right) .
\end{gathered}
$$

The mass flow in the second half period follows by symmetry. Assuming that the mass transport is small, we can neglect the change in ripple size during one half cycle, and add the contributions from each half period.

To obtain a closed set of dynamical equations we have to establish how the lengths $l_{i}$ and $r_{i}$ evolve under a certain mass flow. When an amount of mass $M$ is deposited on the right slope of ripple $i$, we incorporate this by an increase of $l_{i}$ and a subsequent decrease of $l_{i+1}$; the length $r_{i}$ itself does not change. Assuming for simplicity the angle of repose to be $45^{\circ}$, we can calculate the volume of the slab of deposited sand and find that the change in the length of nearby ripples is

$$
\begin{gathered}
\Delta l_{i}=\Delta m_{r i} /\left(2 r_{i}\right), \\
\Delta l_{i+1}=-\Delta m_{r i} /\left(2 r_{i}\right) .
\end{gathered}
$$

Ignoring higher order effects we obtain the total change in the length as a function of the mass changes as

$$
\begin{gathered}
\Delta r_{i}=-\frac{\Delta m_{l i+1}}{2 l_{i+1}}+\frac{\Delta m_{l i}}{2 l_{i}}, \\
\Delta l_{i}=\frac{\Delta m_{r i}}{2 r_{i}}-\frac{\Delta m_{r i-1}}{2 r_{i-1}} .
\end{gathered}
$$

This relation together with the mass flow from Eq. (8) defines the refined model. This model has the same linear stability properties as the minimal model defined in Eq. (5).

The total length of the system is conserved, and the total mass is approximately conserved. The masses that are ignored are associated with the small areas that are crosshatched in Fig. 6. It is possible to formulate the model in a strictly mass-conserving manner, by updating the slope lengths when both removing and depositing mass, but this does not alter the model in any substantial way.

In our numerical simulations two different forms of the mass transport function $f(\lambda)$ were used. Both functions have a maximum at $\lambda=\lambda_{\text {min }}=1 / 2$ and are zero at 0 and $\lambda_{\text {max }}$. The simplest function that satisfies these requirements is bi linear, while a smooth function with a quadratic maximum that satisfies $f(0)=f\left(\lambda_{\text {max }}\right)=0$ can be constructed as the sum of a linear function and a square root (see Fig. 7)

$$
\begin{aligned}
f(z)= & \frac{4 z}{2-\lambda_{\max }}+\frac{\lambda_{\max }\left(\lambda_{\max }-4\right)}{2\left(\lambda_{\max }-2\right)^{2}} \\
& +\frac{\lambda_{\max }}{2\left(\lambda_{\max }-2\right)^{2}} \sqrt{16\left(\lambda_{\max }-2\right) z+\left(\lambda_{\max }-4\right)^{2}} .
\end{aligned}
$$

This smooth function for $\lambda_{\max }=1.6$ resembles the one found from the computational flow model in Sec. II B.

\section{Coarsening of fully developed ripples}

When ripples are grown experimentally from a flat bed, initially many small ripples are created. They subsequently 


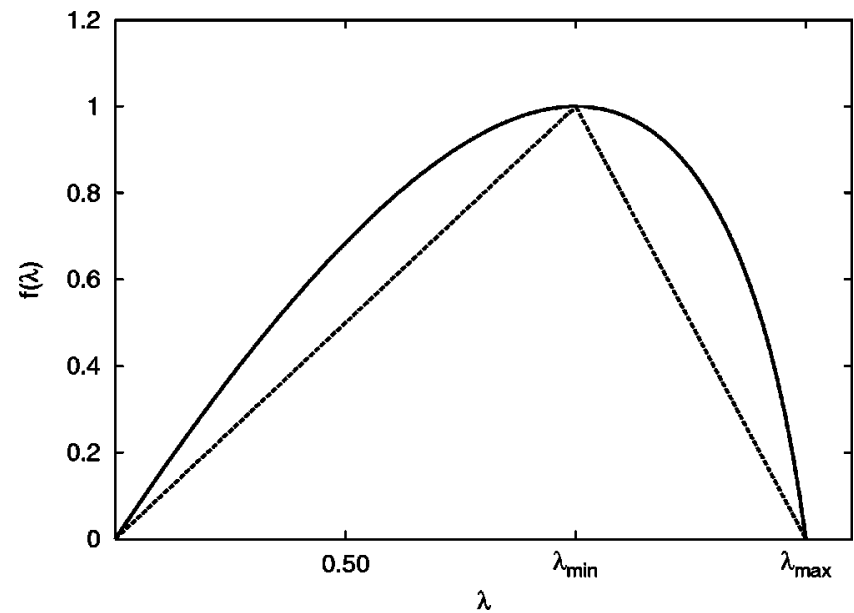

FIG. 7. The interaction functions used in the models. Smooth function, (full line) and the bilinear function (broken line), for a value of $\lambda_{\max }=1.5$.

coarsen and form a final regular steady state with a welldefined final wavelength (see for example Fig. 1 in [19]). Our model shows the same behavior for initial conditions of (disordered) unstable small wavelength patterns. An example of such evolution is shown in Fig. 8. A fast coarsening process is seen in the beginning $(t<1)$, followed by a slower relaxation toward an equilibrium state. The important dynamical process leading to the equilibrium state is the annihilation of ripples, with each annihilation resulting in a longer average ripple length; creation does not play a role here. After the final annihilation, slow diffusive dynamics sets in.

The stability analysis performed in Sec. III B shows that a wide range of ripple wave lengths can be linearly stable, namely $\lambda_{\min }<\lambda<\lambda_{\max }$. We will show here that, starting from small ripples, the dynamics leads to the selection of a sharply defined final wavelength. We assume periodic boundary conditions in our simulations. The parameters entering the model are the length of the domain $L$ and the maximum ripple length $\lambda_{\max }$. The initial conditions are disordered ripples with an average wavelength $\lambda_{0}<\lambda_{\min }$.

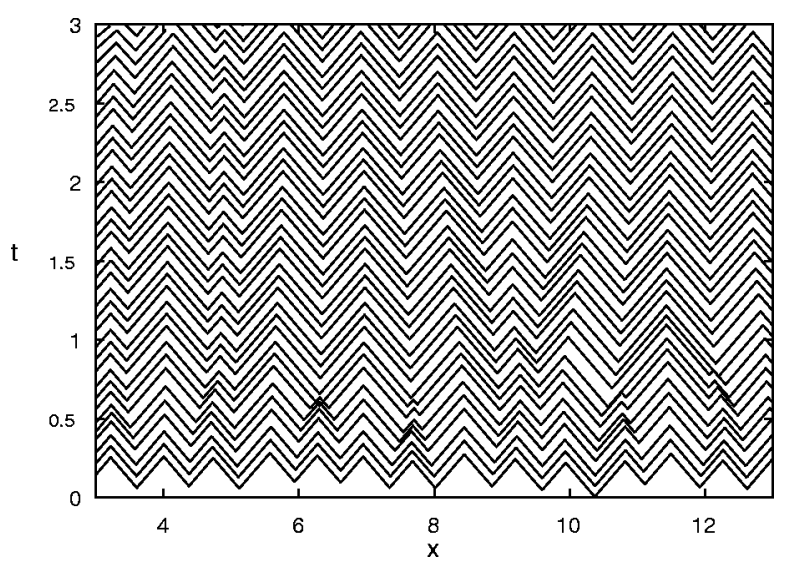

FIG. 8. An example of the dynamics of the model using the linear interaction function and $\lambda_{\max }=1.35$ The time scale is arbitrary.

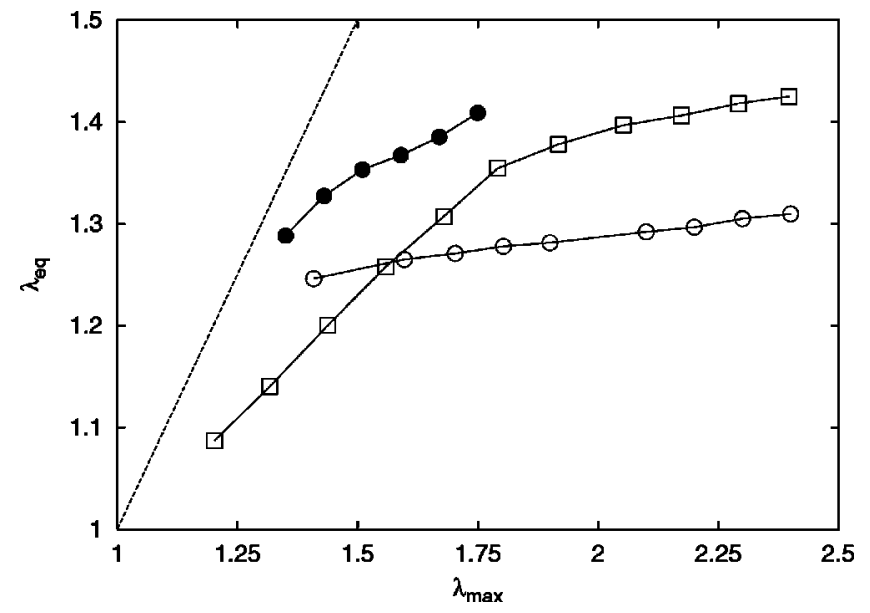

FIG. 9. The equilibrium wavelength as a function of $\lambda_{\max }$. The squares correspond to results obtained using the bilinear interaction function, the open circles correspond to the smooth function and the filled circles correspond to the two-dimensional (2D) model. The dashed line indicates the maximum possible wavelength $\lambda=\lambda_{\max }$. The initial number of ripples were 1200 and the initial ripple length was $0.7 \pm 0.05$.

We found that the final wavelength is quite independent upon the initial average wavelength $\lambda_{0}$ (when this is sufficiently small) and the initial degree of disorder. This result could not have been predicted a priori from the model equations, but is in good agreement with experimental evidence.

The final wavelength does, however, depend on the shape of the interaction function and the value of $\lambda_{\max }$. In Fig. 9, $\lambda_{e q}$ is plotted as a function of $\lambda_{\max }$ for the two interaction functions. The final wavelength appears to be a nontrivial function of $\lambda_{\max }$ for both interaction functions. The interaction function that resembles the one from the numerical flow calculations (the smooth function with $\lambda_{\max }=1.6$ ) results in an equilibrium wavelength of $\lambda_{e q}=1.28 \pm 0.03$, a result that is in good agreement with ripple lengths measured in experiments.

\section{TWO-DIMENSIONAL RIPPLE PATTERNS}

Unless one forces the ripple patterns to occur in a narrow channel or annulus, ripple patterns are two dimensional, even though the alignment perpendicular to the flow yields quasione-dimensional patterns. However, during the evolution toward the final state the pattern contains many defects [4]. We have thus extended our model to study their role in the selection of the final state.

In our two-dimensional model the individual rows of ripples are similar to those in Fig. 6 and are labeled by indices $i$ and $j$, where $j$ is the new coordinate perpendicular to the driving direction. In the $i$ direction, the mass flow is given by expression (8). We then determine which ripples are neighbors in the $j$ direction, and impose an angle of repose in the $j$ direction by inducing a flow of mass between ripple $j$ and $j+1$ when their height difference is above a certain maximum. At a defect, such mass flow can nucleate a new ripple in the trough of an adjacent row. Finally, it is reasonable to expect that a $j$ flow is induced when the ripples are not 

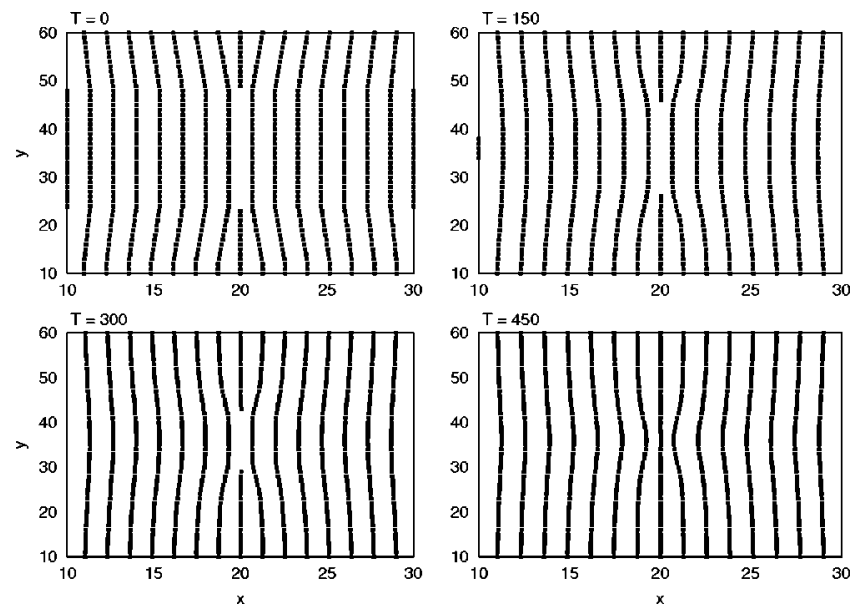

FIG. 10. An example of a climbing defect in a system with 30 ripples in the middle and 31 ripples at the top and bottom. $\left(\lambda_{1}\right.$ $=1.29, \lambda_{2}=1.33$ ).

aligned perpendicular to the main flow, and the simplest choice for such flow between ripple $j$ and $j+1$ is $-\Delta m_{l j}$ $=C_{x}\left(x_{j+1}-x_{j}\right)$. The coupling in the $j$ direction is thus diffusive, and basically acts to align the ripple crests perpendicular to the oscillatory motion. In the simulations that are presented, the value of $C_{x}$ has been fixed to 0.08 . The qualitative results are not sensitive to a change of this parameters.

To study the motion of defects in this model, we initiate the system with two patches of nearby wavelengths $\lambda_{1}$ and $\lambda_{2}$ separated by two defects. The motion of these defects depends on the values of the wavelengths (Fig. 10), and we find that when these are larger than 1.28 0.02 the defect climbs in the direction of the lowest wavelength, otherwise it moves in the direction of the largest wavelength. Thus in a pattern with many defects, as encountered during the coarsening process, one expects that only regions with wavelengths larger than $\lambda_{\text {def }}=1.28$ will survive. We can therefore expect the final wavelength $\lambda_{e q}$ in a two-dimensional system to lie between $\lambda_{\text {def }}$ and $\lambda_{\text {max }}$.

To check this we have performed simulations in large systems with initial conditions consisting of unstable ripple patterns of wavelength 0.5 . The system rapidly coarsens and evolves to a state where most of the wavelengths lie in the 1D stable regime $\lambda_{\text {min }}<\lambda<\lambda_{\text {max }}$. The dynamics then slows down dramatically and becomes dominated by defect climbing. In the final relaxed state of the system the peak of the distribution of ripples lengths lie between $\lambda_{\text {def }}$ and $\lambda_{\max }$. Runs with different values of $\lambda_{\max }$ have confirmed that the equilibrium wavelength in the two-dimensional model is sys- tematically larger than in the one-dimensional case (see Fig. 9).

\section{DISCUSSION AND CONCLUSION}

By focusing on a realistic law for the mass exchange between adjacent ripples, simple models have been formulated that capture a number of phenomena observed in real ripples. First of all, our model predicts the existence of a finite band of stable ripple wave numbers $\lambda_{\min }<\lambda<\lambda_{\max }$, which is consistent with the hysteresis observed in experiments $[7,29]$. The model predicts that the instabilities encountered once these boundaries are crossed are of short wavelength nature, in agreement with experiments on one- and twodimensional sand patterns performed in Copenhagen [4].

Coarsening that occurs in the intermediate stages of the development of vortex ripples is present in our models, and we predict that even though there is a finite band of stable ripple patterns, the dynamics selects a well defined final wave length. The exact value of the final wave length depends on the details of the mass exchange function, however for a function similar to the results from our simulations of the fluid and sand flow, we find an equilibrium wavelength of $\lambda=1.28$.

A good collapse of the mass exchange function with the maximum shear stress $\theta_{\max }$ indicates that the final wave number should be independent of $\theta_{\max }$ as long as suspension is not important.

Following the picture of the mass exchange as in the models, it is clear that the maximum value of the mass exchange function sets a time scale for the evolution of the ripples. We have shown that this maximum value is approximately proportional to $\theta_{\max }^{1.5}$. Thus the time scale of the evolution of the ripples can be expected to scale as $\theta_{\max }^{1.5}$.

Finally we have demonstrated that the models can be applied to two-dimensional ripple patterns, and have found that defect motion renders the final wavelength of ripples in two dimensions larger than in one dimension.

All these predictions are open to experimental verification. In particular, we are eager to see how consistent the mass exchange mechanism proposed here is for real data of ripple evolution.

\section{ACKNOWLEDGMENTS}

It is a pleasure to acknowledge discussions with Markus Abel, Tomas Bohr, Jørgen Fredsøe, Jonas Lundbek Hansen, Nigel Marsh, and Alexandre Stegner. M.v.H. acknowledges financial support from the EU under Contract No. ERBFMBICT 972554. M.-L.C. thanks the Niels Bohr Institute for hospitality.
[1] R. A. Bagnold, The Physics of Blown Sand and Desert Dunes (Chapman and Hall, Methuen, London, 1941).

[2] A. J. Raudkivi, J. Hydraul. Eng. 123(1), 58 (1997).

[3] R. A. Bagnold, Proc. R. Soc. London, Ser. A 187, 1 (1946).

[4] J. L. Hansen, M. v. Hecke, A. Haaning, C. Ellegaard, K. H.
Andersen, T. Bohr, and T. Sams, Nature (London) 410, 324 (2001).

[5] K. H. Andersen, Phys. Fluids 13, 58 (2001).

[6] H. Nishimori, M. Yamasaki, and K. H. Andersen. Int. J. Mod. Phys. B 12, 257 (1998). 
[7] K. E. B. Lofquist, in Proceedings of the 17th International Conference on Coastal Engineering in Sydney, Australia, 1980 (unpublished).

[8] J. F. A. Sleath, Cont. Shelf Res. 1, 33 (1982).

[9] P. P. Mathisen and O. S. Madsen, J. Geophys. Res. [Oceans] 101(C7), 16533 (1996).

[10] J. Fredsøe, K. H. Andersen, and M. Sumer, Coastal Eng. 38, 177 (1999).

[11] K. H. Andersen and M. Lohmann, in Proceedings of the International Conference on Coastal Engineering, Sydney, Australia, 2000 (unpublished).

[12] E. A. Hansen, J. Fredsøe, and R. Deigaard, J. Waterway, Port, Coastal, Ocean Eng. 120, 37 (1994).

[13] R. A. Anderson, Earth-Sci. Rev. 29, 77 (1990).

[14] H. Nishimori and N. Ouchi, Phys. Rev. Lett. 71, 197 (1993).

[15] L. Prigozhin, Phys. Rev. E 60, 729 (1999).

[16] O. Terzidis, P. Claudin and J.-P. Bouchaud, Eur. Phys. J., 5, 245 (1998).

[17] Z. Csahók, C. Misbah, and F. A. Valance, Physica D 128, 87 (1999).

[18] A. Betat, V. Frette, and I. Rehberg, Phys. Rev. Lett. 831, 88 (1999)

[19] A. Stegner and J. E. Wesfreid, Phys. Rev. E 60, R3487 (1999).

[20] M. A. Scherer, F. Melo, and M. Marder, Phys. Fluids 11, 58 (1999).

[21] P. Blondeaux, J. Fluid Mech. 218, 1 (1990).
[22] P. Blondeaux and G. Vittori, J. Fluid Mech. 226, 257 (1991).

[23] G. Vittori and P. Blondeaux, J. Fluid Mech. 239, 23 (1991).

[24] E. Foti and P. Blondeaux, Coastal Eng. 25, 227 (1995).

[25] H. Ayrton, Proc. R. Soc. London, Ser. A 84, 285 (1910).

[26] J. R. Dingler, PhD thesis, University of California (1975).

[27] P. Nielsen, J. Geophys. Res., [Oceans] 86, 6467 (1981).

[28] P. L. Wiberg and C. K. Harris, J. Geophys. Res., [Oceans] 99, 775 (1994).

[29] P. Traykovski, A. E. Hay, J. D. Irish, and J. F. Lynch, J. Geophys. Res., [Oceans] 104, 1505 (1999).

[30] P. Nielsen, Ph.D. thesis, Danish Technical University (1979).

[31] Landau and Lifshitz, Fluid Mechanics (Pergamon Press, New York, 1963).

[32] J. Fredsøe and R. Deigaard, Mechanics of Coastal Sediment Transport (World Scientific, Singapore, 1992).

[33] E. Meyer-Peter and R. Müller, in Proceedings of Second Meeting of International Association for Hydraulic Structures Research, Stockholm, 1948 (unpublished).

[34] F. Engelund and J. Fredsøe, Nord. Hydrol. 7, 293 (1976).

[35] A. Kovacs and G. Parker, J. Fluid Mech. 267, 153 (1994).

[36] D. C. Wilcox, AIAA J. 26, 1299 (1988).

[37] K. H. Andersen, Ph.D. thesis, Copenhagen University, 1999, http://www.nbi.dk/ kenand/Thesis.html.

[38] There is some experimental evidence that in some parameter regime, possibly due to next-nearest-neighbor interactions, such period 2 states can become stable [20]. 